\title{
Mechanism of strain release in carbon nanotubes
}

\author{
Marco Buongiorno Nardelli, B. I. Yakobson, and J. Bernholc \\ Department of Physics, North Carolina State University, Raleigh, North Carolina 27695-8202
}

(Received 14 October 1997)

\begin{abstract}
Static and dynamical properties of carbon nanotubes under uniaxial tension have been investigated via quantum and classical simulations. In strained nanotubes at high temperatures we observe the spontaneous formation of double pentagon-heptagon defect pairs. Tubes containing these defects are energetically preferred to uniformly stretched tubes at strains greater than 5\%. These topological defects act as nucleation centers for the formation of dislocations in the originally ideal graphite network, and they constitute the onset of a plastic deformation of the carbon nanotube. The mechanism of formation of such defects, their energetics, and transformations are described. [S0163-1829(98)50208-1]
\end{abstract}

Since their discovery in $1991,{ }^{1}$ carbon nanotubes have attracted much interest due to their peculiar character at a crossroad between traditional carbon fibers and fullerenes. They hold substantial promise for use as superstrong fibers, catalysts, and as components of novel electronic devices. Despite the potential impact that new composites based on carbon nanotubes would have in many areas of science and industry, very little is known about the microscopic origin of their strength and a complete theoretical understanding of their behavior is desirable. The excellent resistance of carbon nanotubes to bending has already been observed experimentally and studied theoretically. ${ }^{2-4}$ The remarkable flexibility of the hexagonal network allows the system to sustain very high bending angles, kinks, and highly strained regions. In addition, nanotubes are observed to be extremely resilient, suggesting that even largely distorted configurations (axial compression, twisting) can be due to elastic deformations with no atomic defects involved. ${ }^{2,3,5,6}$

The present paper is mainly focused on the mechanism of strain release in armchair carbon nanotubes under uniaxial tension. Via energetic relaxations and dynamical calculations, both using first-principles methods and classical manybody potentials, we have been able to identify the first stages of the mechanical yield of carbon nanotubes. Beyond a critical value of the tension, the system releases its excess strain via a spontaneous formation of topological defects. The first defect to form corresponds to a $90 \mathrm{deg}$ rotation of a $\mathrm{C}-\mathrm{C}$ bond about its center, the so-called Stone-Wales transformation, ${ }^{7}$ which produces two pentagons and two heptagons coupled in pairs (5-7-7-5). Static calculations under fixed dilation show a crossover in the stability of this defect configuration with respect to the ideal hexagonal network. The crossover is observed at about $5 \%$ tensile strain in $(5,5)$ and $(10,10)$ armchair tubes. This implies that the (5-7-7-5) defect is effective in releasing the excess strain energy in a tube under tensile strain. Moreover, through its dynamical evolution, this defect acts as a nucleation center for the formation of dislocations in the ideal graphite network and it may cause a possible plastic behavior in carbon nanotubes. ${ }^{8}$

The quantum simulations were carried out in the framework of a multigrid-based density-functional total-energy method that uses a real-space grid as the basis. ${ }^{9}$ The PerdewZunger parametrization of the Ceperley and Alder form ${ }^{10}$ of the exchange and correlation energy was used. Nonlocal, norm-conserving pseudopotentials ${ }^{11}$ were included using the Kleinman and Bylander approach. ${ }^{12} \mathrm{~A}$ tetragonal supercell with grid spacing of 0.38 a.u. in the $x, y$, and $z$ directions was employed after a careful convergence study, thereby ensuring that the real-space description of the pseudopotential is accurate. A large vacuum region of $\sim 6 \AA$ surrounded the tube in order to avoid interactions between its periodic images, while periodic boundary conditions were imposed along the axis of the tube itself. All the quantum simulations have been done using a $(5,5)$ armchair nanotube in a supercell of length $l=14.75 \AA$ at zero strain, which included 120 carbon atoms. The same geometry has also been used in the classical simulations in order to investigate the reliability of the latter.

In the classical simulations, carbon atoms were modeled with a classical many-body Tersoff-Brenner potential. ${ }^{13}$ This potential accurately reproduces the lattice constants, binding energies and the elastic constants of both graphite and diamond. A standard fifth-order Beeman-Verlet algorithm was used to integrate the equations of motion with a basic time step of $0.5 \mathrm{fs}$. The Anderson temperature-scaling algorithm was used for the temperature control. Minimum energy configurations in zero temperature calculations were obtained via the conjugate gradient algorithm. Due to the smaller computational workload, supercells with up to 480 atoms have been used in the classical simulations. The convergence of the total-energy results and of the dynamical behavior with respect to supercell size has also been carefully investigated.

Quantum molecular dynamics simulations have been performed in the canonical ensemble on a $(5,5)$ tube under $10 \%$ tensile strain. The ideal nanotube has been initially relaxed in the strained geometry and subsequently heated to $1800 \mathrm{~K}$. Multiple Nose-Hoover thermostats have been used to control the temperature during the simulation. ${ }^{14}$ All the simulations were carried out on the massively parallel Cray T3D supercomputer with a time step of 0.64 fs for an overall time of $\sim 6$ ps. After an initial equilibration at $1800 \mathrm{~K}(\sim 1 \mathrm{ps})$, a first precursor of a mechanical instability appears. A single $\mathrm{C}-\mathrm{C}$ bond rotation generates a double pentagon-heptagon defect (5-7-7-5), as shown in Fig. 1. The whole process of bond rotation, which leads to the breaking and rebonding of a $\mathrm{C}-\mathrm{C}$ 
a)

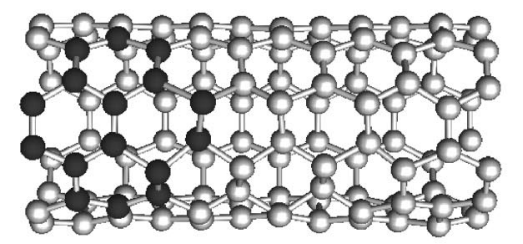

b)

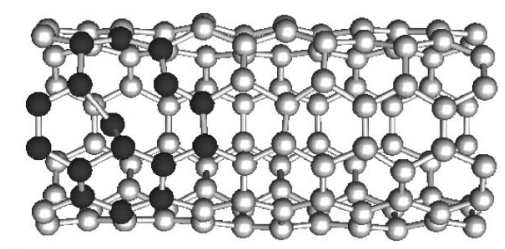

c)

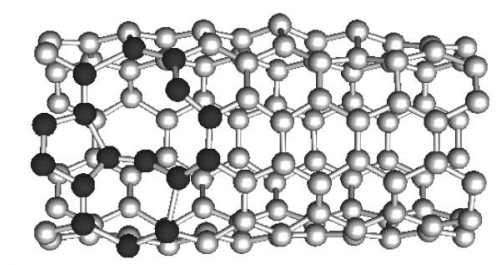

d)

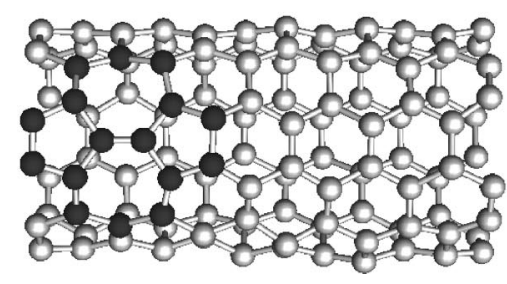

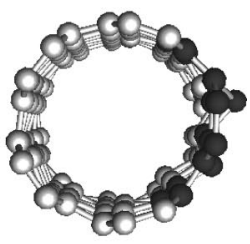
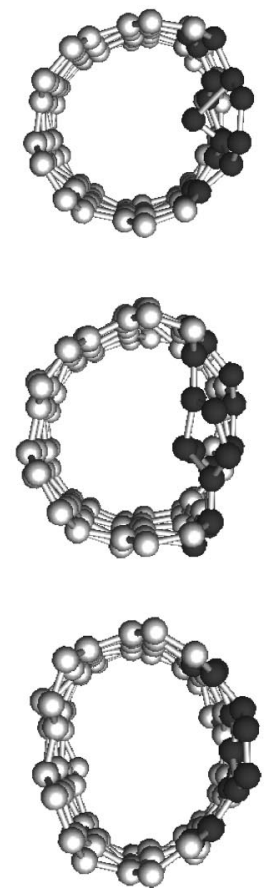

FIG. 1. Kinetic mechanism of (5-7-7-5) defect formation from an $a b$ initio quantum mechanical molecular-dynamics simulation for the $(5,5)$ tube at $1800 \mathrm{~K}$. The atoms that take part in the StoneWales transformation are highlighted in black. The four snapshots show the various stages of the defect formation: (a) system in the ideal configurations $(\mathrm{t}=0.00 \mathrm{ps})$; $(\mathrm{b})$ breaking of the first bond $(\mathrm{t}$ $=0.10 \mathrm{ps})$; (c) breaking of the second bond $(\mathrm{t}=0.15)$; (d) the defect is formed $(\mathrm{t}=0.20 \mathrm{ps})$.

bond, takes about $0.2 \mathrm{ps}$. This topological change is stable during the remaining simulation time $(\sim 1 \mathrm{ps})$. The energetics of this defect has been investigated via zero temperature calculations. Both the equilibrium and the $10 \%$ strained tubes are (meta-)stable in the ideal graphite structure and in the presence of the (5-7-7-5) defect. At zero strain, the energy of the defect configuration is higher than the ideal one by $2.34 \mathrm{eV}$. However, in the $10 \%$ strained tube, the energy of the structure with the defect is lower than the ideal one by $-1.77 \mathrm{eV}$. This is because this defect is very effective in reducing the tensile strain, since the two heptagons can be stretched more than the hexagons while keeping a $\mathrm{C}-\mathrm{C}$ bond length that is close to the ideal one.

Although very accurate, ab initio simulations suffer from substantial limitations with regard to simulation time and thus to the number of cases that can be studied within our available computer time. To overcome these limitations we examined the description of carbon nanotubes obtained using classical many-body potentials. In order to investigate the reliability of these potentials we first calculated the energy differences between the defect and the ideal structures at the

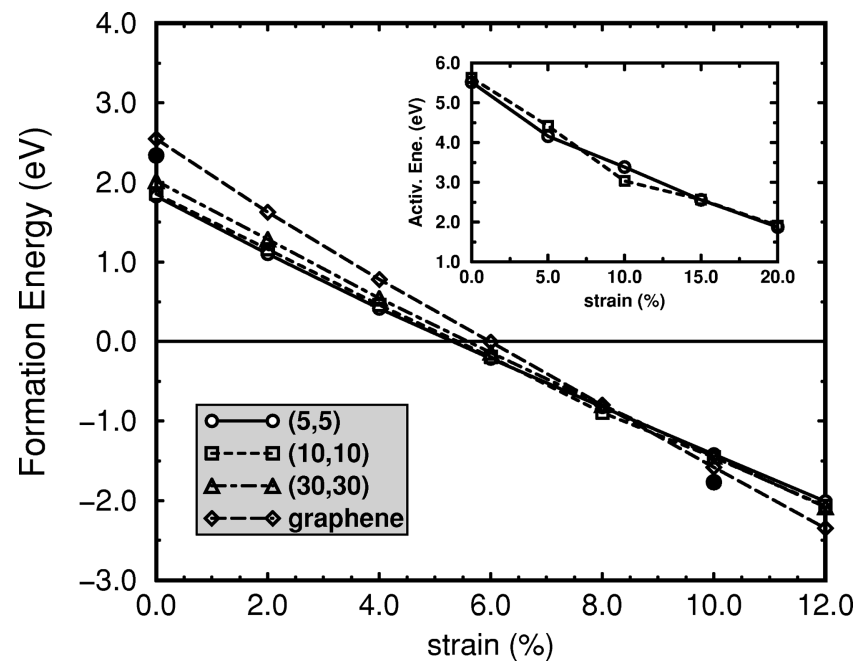

FIG. 2. Formation energy of a pentagon-heptagon defect in various structures vs uniaxial strain. Solid dots are the $a b$ initio results calculated for the $(5,5)$ tube. The inset shows the variation of the activation energy with strain for the $(5,5)$ and $(10,10)$ tubes. See text.

equilibrium length and at the $10 \%$ tensile strain for the same $(5,5)$ nanotube. Both the energies and the relaxed atomic coordinates agree quite well with the $a b$ initio results: we obtain $+1.84 \mathrm{eV}$ and $-1.42 \mathrm{eV}$ for the energy differences in the equilibrium and the strained structures, compared to the $a b$ initio ones of +2.34 and $-1.77 \mathrm{eV}$, respectively. Moreover, we observe a very similar dynamical behavior for the (5-7-7-5) defect formation in both the approaches. Despite minor quantitative differences, the agreement between the two different methods is thus very good, both in the dynamics and in the atomic relaxations. This agreement gives us the opportunity to explore a much larger range of simulation time and to obtain a more general description of the mechanical behavior of carbon nanotubes.

In Fig. 2 we show the results of classical potential calculations of the formation energy of a single (5-7-7-5) defect as a function of uniaxial strain in three armchair tubes, $(5,5)$, $(10,10),(30,30)$, and of a single sheet of graphite (graphene). In the case of the graphene sheet the lattice constant in the direction perpendicular to the strain was minimized, thus accounting for the Poisson ratio. The defect structure starts to be energetically favored after about $5 \%$ tensile strain in the tubes, and somewhat later in graphene, due to the absence of the additional strain induced by the curvature of the tubular structure. The $a b$ initio values for the formation energy of the defect in the $(5,5)$ tube are also shown. Our results at zero strain are in agreement with those of a previous theoretical study of defects in large fullerene structures. ${ }^{15}$

The simplest kinetic pathway that produces the double pentagon-heptagon pair is a rotation of a C-C bond by $90 \mathrm{deg}$ about its center. The activation energies for the rotation that produces the (5-7-7-5) defect has been calculated for the $(5,5)$ and $(10,10)$ tubes using the Tersoff-Brenner potential, and are summarized in Table I and in the inset of Fig. 2. We obtained the barriers by changing the angle of the plane of the rotating $\mathrm{C}-\mathrm{C}$ bond and relaxing all the remaining internal coordinates of the tube at each step. As expected, the potential energy barrier decreases with the strain. At equilibrium 
TABLE I. Energy barriers for bond rotation in the $(5,5)$ and $(10,10)$ tubes.

\begin{tabular}{lcc}
\hline \hline \% strain & $\Delta E(5,5)(\mathrm{eV})$ & $\Delta E(10,10)$ \\
0 & 5.52 & 5.63 \\
5 & 4.16 & 4.42 \\
10 & 3.39 & 3.04 \\
15 & 2.56 & 2.57 \\
20 & 1.88 & 1.91 \\
\hline \hline
\end{tabular}

length ( $0 \%$ strain) the barrier is smaller in the tubes than in graphene because of the additional tubular strain. Our calculated value for the energy barrier in a flat graphite sheet is $6.16 \mathrm{eV}$, which compares well with a previous theoretical estimate, $\sim 7 \mathrm{eV}$, obtained using the tight-binding method. ${ }^{16}$ The large value of this barrier comes from the need to break strong C-C bonds in the kinetic pathway from the ideal hexagonal rings to the (5-7-7-5) defect. The inset in Fig. 2 shows graphically the variation of the activation energy with strain; in accordance with the Polanyi-Bronsted rule the activation energy decreases with the decreasing formation energy. ${ }^{17}$ The slope of this curve in the initial stage is almost a factor of 2 lower than that of the formation energy. The activation barrier is sizable up to $25-30 \%$ strain, which is consistent with the large breaking strain in high-rate tension simulations. $^{18}$

Under the conditions of our high-temperature classical molecular dynamics simulations the formation of the (5-7$7-5)$ defect in a strained tube is a reversible process. We annealed a $(5,5)$ carbon nanotube under $10 \%$ tensile strain at $2500 \mathrm{~K}$. After a few picoseconds of equilibration a (5-7-7-5) defect was formed. The resulting tube was then returned to its original elongation through a rescaling of the atomic coordinates and let evolve for a few picoseconds. The system readily recovered the original hexagonal network. The same behavior was observed in tubes at equilibrium length in which various defects have been artificially introduced and annealed at sufficiently high temperatures. This is a clear signature of the remarkable resilience and extreme flexibility of these nanostructures, which show a ductile rather than brittle behavior for the $10 \%$ elongation considered in this paper.

The knowledge of the activation energy of the (5-7-7-5) defect allows for a rough estimate of the rate of defect formation in a tube under different temperature and strain conditions. Using a simple Arrhenius expression, the bond rotation rate is given by $\Gamma \propto N_{\text {bonds }} \bar{\nu} \exp \left(-\Delta E / k_{B} T\right)$, where $\Delta E$ is the activation energy for the bond rotation, ${ }^{19} T$ is the absolute temperature, $k_{B}$ is the Boltzmann constant, $N_{\text {bonds }}$ is the number of bonds, and $\bar{\nu}$ is an effective frequency associated with the vibration of the bond in the direction of the saddle point. In our case we estimate the latter using the frequency of the bond rotation mode in a carbon nanotube $\left(\sim 10^{13} \mathrm{sec}^{-1}\right) .{ }^{20}$ With this proviso and the calculated values for the activation energies, we find that at room temperature the tubes are very stable in their hexagonal equilibrium configuration, since the exponential factor is extremely small. On the other hand, at higher temperatures this scenario is changed. In the same tube as above but at $2000 \mathrm{~K}$, the rate of defect formation raises to $10^{7} \mathrm{sec}^{-1} /$ bond. This is in a)

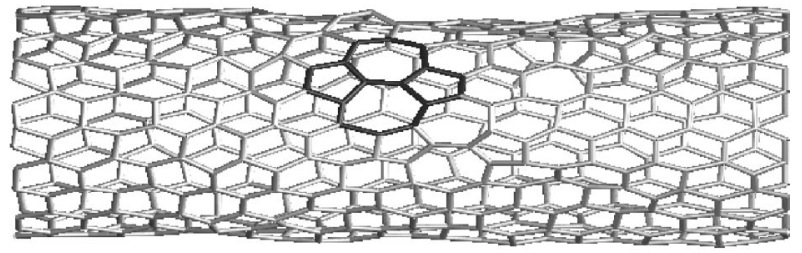

b)

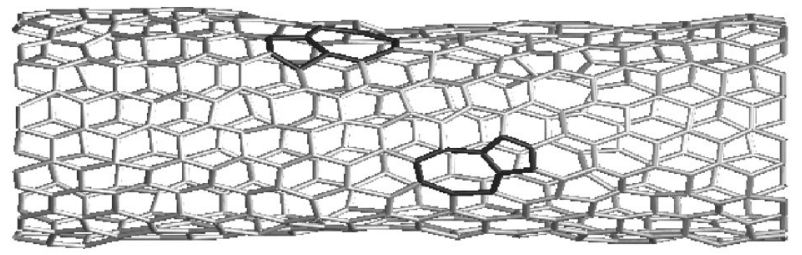

c)

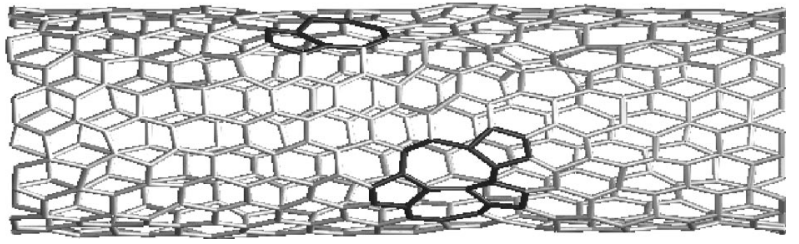

FIG. 3. Time evolution of a particular (5-7-7-5) defect from a classical simulation for a $(10,10)$ carbon nanotube at $2000 \mathrm{~K}$ under $10 \%$ uniaxial strain. The defect structure is highlighted in black. (a) Formation of the pentagon-heptagon defect whose evolution in time will be followed $(t=1.5 \mathrm{~ns})$. (b) The defect splits and starts diffusing $(\mathrm{t}=1.6 \mathrm{~ns})$, note that a $(10,10) /(10,9) /(10,10)$ heterojunction has formed; (c) Another bond rotation has led to the formation of a (5-7-5-8-5) defect $(\mathrm{t}=2.3 \mathrm{~ns})$.

agreement with the rate observed in our dynamic simulations. Moreover, the formation energies of these defects are quite low, indicating that they can readily form at growth temperatures. Indeed, kinks and changes in diameter have been frequently observed in multiwalled tubes, which are grown at temperatures exceeding $3000 \mathrm{~K}$. For a material at thermal equilibrium, the number of defects of a particular type is given by $\Gamma \propto N_{\text {sites }} \exp \left(-E_{f} / k_{B} T\right)$, where $\mathrm{E}_{f}$ is the formation energy ${ }^{19}$ of the defect that for tubes and fullerene structures in general does not exceed a few eV. ${ }^{15}$ Even for single-walled tubes, which are grown at $\sim 1200 \mathrm{C}$, the above estimate suggests that there may be a point defect every few tenths of a mm, and these defects can act as nucleation centers for deformation of the system under tensile strain.

A better understanding of the kinetics of deformation of a carbon nanotube under tensile strain conditions can be gleaned from long-time classical molecular-dynamics simulations on larger tubes. For this reason, we studied the time evolution of a relatively long fragment ( $3 \mathrm{~nm}, 480$ atoms) of a $(10,10)$ tube at $2000 \mathrm{~K}$ under $10 \%$ axial strain. After an initial annealing to $2000 \mathrm{~K}$, with a rate of temperature increase of $50 \mathrm{~K}$ per ps, the system was evolved for $2.5 \mathrm{~ns}$. Within the first $1.1 \mathrm{~ns}$ a few (5-7-7-5) defects are formed in the tube and each of them remains localized in the region where they have been formed. After $1.5 \mathrm{~ns}$ another (5-7-7-5) defect is formed [see Fig. 3(a)], which shows a peculiar time evolution. After 100 ps from its formation, the two pentagonheptagon pairs split, and eventually one of them starts dif- 
fusing within the helical structure of the tube [Fig. 3(b)], while the other remains trapped in the original position by an additional (5-7-7-5) defect. After another $350 \mathrm{ps}$, the 5-7 defect that migrated from its original position transforms into another topological defect: a (5-7-5-8-5), i.e., an octagon and a pair of pentagons are added to the original pentagonheptagon pair [Fig. 3(c)]. The introduction of topological defects can change the index of nanotubes. The 5-7 defect is the smallest defect that can change the indices (or the chirality in chiral tubes) without drastically altering the local curvature of the nanotube. ${ }^{21-24}$ What we observe during the simulation is actually a chirality change within the $(10,10)$ tube, and a strain-induced spontaneous formation of a $(10,10) /(10,9) /(10,10)$ heterojunction. This is produced by the splitting of the two pentagon-heptagon pairs comprising the original defect. It is important to note that a (5-7-7-5) defect does not introduce any index change, and neither does the addition of an octagon and a pair of pentagons to the pre-existing pentagon-heptagon pair, as in the last part of our simulation. The unique electronic and transport properties of heterostructures formed by joining carbon nanotubes of different indices ${ }^{21,23-25}$ and of bond-rotation defects ${ }^{26}$ have already been extensively investigated.

From a topological point of view, the splitting of the (57-7-5) defect produces a remarkable effect: ${ }^{8}$ the separation and glide of 5-7 dislocations changes the chirality of the nanotube and leads to the formation of heterojunctions.

In summary, $a b$ initio and classical simulations show that carbon nanotubes indeed have outstanding mechanical properties with respect to tensile dilation. For deformations larger than $5 \%$, the system finds natural release of the excess strain via a reversible formation of topological defects, the simplest of them being the double pentagon-heptagon pair. These defects act as nucleation centers for the formation of dislocations in the originally ideal graphite network, and constitute the onset of the possible plastic deformation of a carbon nanotube.

We are pleased to acknowledge fruitful discussions with C. Brabec, E. Briggs, M. Ramamoorthy, M. Wensell, and C. Roland.

${ }^{1}$ S. Iijima, Nature (London) 354, 56 (1991).

${ }^{2}$ S. Iijima, C. Brabec, A. Maiti, and J. Bernholc, J. Chem. Phys. 104, 2089 (1996).

${ }^{3}$ B. I. Yakobson, C. J. Brabec, and J. Bernholc, Phys. Rev. Lett. 76, 2511 (1996).

${ }^{4}$ M. R. Falvo, G. J. Clary, R. M. Taylor II, V. Chi, F. P. Brooks, Jr., S. Washburn, and R. Superfine, Nature (London) 389, 582 (1997).

${ }^{5}$ J. Despres, E. Daguerre, and K. Lafdi, Carbon 33, 87 (1995).

${ }^{6}$ N. Chopra, L. Benedict, V. Crespi, M. L. Cohen, S. G. Louie, and A. Zettl, Nature (London) 377, 135 (1995); R. Ruoff and D. Lorents, Bull. Am. Phys. Soc. 40, 173 (1995).

${ }^{7}$ A. J. Stone and D. J. Wales, Chem. Phys. Lett. 128, 501 (1986).

${ }^{8}$ B. I. Yakobson (unpublished).

${ }^{9}$ E. L. Briggs, D. J. Sullivan, and J. Bernholc, Phys. Rev. B 52, R5471 (1995); ibid. 54, 14362 (1996).

${ }^{10}$ D. Ceperley and B. Alder, Phys. Rev. Lett. 45, 566 (1980).

${ }^{11}$ G. Bachelet, D. Hamann, and M. Schlüter, Phys. Rev. B 26, 4199 (1982); D. Hamann, M. Schlüter, and C. Chiang, Phys. Rev. Lett. 43, 1494 (1979); D. Hamann, Phys. Rev. B 40, 2980 (1989).

${ }^{12}$ L. Kleinman and D. Bylander, Phys. Rev. Lett. 48, 1425 (1982).

${ }^{13}$ J. Tersoff, Phys. Rev. Lett. 61, 2879 (1988); Phys. Rev. B 37, 6991 (1988); D. W. Brenner, ibid. 42, 9458 (1990).

${ }^{14}$ S. Nosé, Mol. Phys. 52, 255 (1984); W. G. Hoover, Phys. Rev. A 31, 1695 (1985); G. J. Martyna, M. L. Klein, and M. Tuckerman, J. Chem. Phys. 97, 2635 (1992).

${ }^{15}$ C. J. Brabec, A. Maiti, and J. Bernholc, Chem. Phys. Lett. 219 , 473 (1994). Estimates of the formation energy for the Stone-

Wales defect in various graphitic structures were also obtained by M. Terrones and H. Terrones, Fullerene Sci. Technol. 4, 517 (1996). The values of Ref. 27 are substantially higher than the results of both our quantum and classical calculations.

${ }^{16}$ V. H. Crespi, L. X. Benedict, M. L. Cohen, and S. G. Louie, Phys. Rev. B 53, R13 303 (1996).

${ }^{17}$ E. A. Moelwyn-Hughes, Physical Chemistry (Pergamon, New York, 1961), p. 1265.

${ }^{18}$ B. I. Yakobson, M. P. Campbell, C. J. Brabec, and J. Bernholc, Comput. Mater. Sci. 8, 341 (1997); B. I. Yakobson, M. Buongiorno Nardelli, M. P. Campbell, C. J. Brabec, and J. Bernholc (unpublished).

${ }^{19}$ The complete expression contains the activation (formation) enthalpy of the defect in the exponent, since we study systems under fixed strain conditions. However, both the entropic and the pressure/volume contributions are usually a small part of the enthalpy even at high temperatures, and one can use the internal energy of activation (formation) to obtain a lower bound.

${ }^{20}$ J. Yu, R. K. Kalia, and P. Vashishta, J. Chem. Phys. 103, 6697 (1995).

${ }^{21}$ B. I. Dunlap, Phys. Rev. B 46, 1933 (1992).

${ }^{22}$ T. W. Ebbesen and T. Takada, Carbon 33, 973 (1995).

${ }^{23} \mathrm{Ph}$. Lambin, A. Fonseca, J. P. Vigneron, J. B. Nagy, and A. A. Lucas, Chem. Phys. Lett. 245, 85 (1995).

${ }^{24}$ L. Chico, V. H. Crespi, L. X. Benedict, S. G. Louie, and M. L. Cohen, Phys. Rev. Lett. 76, 971 (1996).

${ }^{25}$ L. Chico, L. X. Benedict, S. G. Louie, and M. L. Cohen, Phys. Rev. B 54, 2600 (1996).

${ }^{26}$ V. H. Crespi, M. L. Cohen, and A. Rubio, Phys. Rev. Lett. 79, 2093 (1997). 